\title{
Encapsulated calcium carbonate suspensions: A drug delivery vehicle sensitive to ultrasound disruption
}

\author{
Brent Lanting*, Joe Barfett*
}

\begin{abstract}
A calcium carbonate suspension, encapsulated within particles of calcium alginate hydrogel, is proposed as a drug delivery device susceptible to ultrasound disruption. Spheres approximately $1 \mathrm{~mm}$ in diameter were prepared by the coaxial airflow method from mixtures of $1 \%$ sodium alginate $(\mathrm{m} / \mathrm{v})$ and each of $50 \%, 75 \%$ and $100 \%$ calcium carbonate $(\mathrm{m} / \mathrm{v})$ in distilled water. This product was subjected to cycles of 85 Watt ultrasound in 1 second on/off bursts via a lab sonication system until fully disintegrated, a process requiring between 8 and 20 minutes depending upon initial calcium carbonate concentrations. The spheres subjected to vortex did not demonstrate any signs of mechanical degeneration after 30 minutes. Before use as a model implant, further work is required to develop a method of drying the particles to make them impermeable to drug diffusion before the time of their disruption with ultrasound.
\end{abstract}

KEYWORDS: alginic acid, microencapsulation, controlled release, ultrasound

\section{INTRODUCTION}

Despite the general use and success of systemic therapies, there has always been an interest in maximizing drug availability in target tissues and minimizing adverse reactions. As a result, many simple, local, and highly effective drug treatments have been developed including drops for eyes and ears, puffers for the respiratory tract, sprays for the sinuses, creams for skin and mucosa, central nervous system shunts and other implants. Local therapy, where appropriate, allows maximum bioavailability in target tissues while minimizing systemic interactions and allowing for the lowering of drug dosages needed for therapeutic effectiveness.

Interest in the development of new drugs is therefore balanced by the increasing emphasis placed on novel

*To whom correspondence should be addressed: Brent Lanting

E-mail: blanting2006@meds.uwo.ca

Joe Barfett

E-mail: jbarfett2006@meds.uwo.ca delivery systems $(1,2)$. Such technologies have been subject to intense development over the past decade and now occupy a multibillion dollar per annum industry in the United States (1). In particular, polymers capable of controlled pharmaceutical release are of significant interest and offer to expand the range of diseases amenable to more localized treatment. The most classic systems rely upon temperature, $\mathrm{pH}$, magnetic fields, light, mechanical force, or ultrasound (as in the presently described system) to cause a change that facilitates drug release by diffusion $(3,4,5,6)$. Such "smart polymers" often work well in vitro but demonstrate severe limitations when constrained to more physiological conditions.

As a simple alternative, we propose a drug delivery vehicle based upon calcium carbonate powder suspended in particles of a calcium alginate hydrogel matrix. Alginate hydrogels are soft and non-toxic polymers traditionally used as food thickeners, but have more recently found applications in both industrial and 
medical encapsulation technologies for controlled release (7). Calcium carbonate powder is insoluble, non-toxic, and known to be susceptible to ultrasound (8). The combination of these two inexpensive materials produces a medically useful hydrogel matrix that can be disintegrated with high intensity sound waves. So far as the authors are aware, this is the first attempt to combine these materials for the purposes of creating an ultrasound activated drug delivery device.

\section{METHODS}

A co-axial airflow encapsulation nozzle was constructed as described by Fiszman et al (7). The authors varied the flow rates described below until macroscopic particles of desirable diameter were attained. It was thought that a $1 \mathrm{~mm}$ spherical diameter offered balance between a small enough object to theoretically inject and yet large enough to work with on a macroscopic scale.

Briefly, a 16 gauge needle, connected to a peristaltic pump via lab tubing, was passed through a $1 / 4$ inch "T" junction and secured in place, thus plugging one of the $\mathrm{T}$ 's three ports. To the horizontal inlet was attached an air hose and pump such that air could be forced around the 90 degree bend and over the needle tip. Via a procedure modified from Yang (9), a $1 \%(\mathrm{~m} / \mathrm{v})$ algin solution was prepared by dissolving $1000.0 \mathrm{mg}$ of sodium alginate (Sigma) in $100.0 \mathrm{cc}$ of distilled water with intense agitation via vortex for $30 \mathrm{~min}$. Preparations were made including $50 \mathrm{~g}, 75 \mathrm{~g}$ and $100 \mathrm{~g}$ of calcium carbonate (Sigma) in the sodium alginate solution to create final $50 \%, 75 \%$ and $100 \%(\mathrm{~m} / \mathrm{v})$ mixtures.

Suspension was forced through the needle by a peristaltic pump at a rate of $8 \mathrm{cc} / \mathrm{min}$ while air flowed around the tip at $4 \mathrm{~L} / \mathrm{min}$, creating enough shear force to convert the liquid stream into fine droplets. These fell $10 \mathrm{~cm}$ into a $4 \%(\mathrm{~m} / \mathrm{v}) \mathrm{CaCl}_{2} \cdot 2 \mathrm{H}_{2} \mathrm{O}$ hardening solution below. Four hours were allowed for full polymerization of the particles before they were spun out of solution by gentle centrifugation and re-suspended in normal saline after three washes. Spheres produced by the above method measured approximately $1 \mathrm{~mm}$ in diameter and were of an opaque white color.

Three particles of each final calcium carbonate concentration were separated into each of three plastic centrifuge tubes and used for the sonication trials. The tubes were subjected to bursts of ultrasound at 85 watts, one second on and one second off, until fully disintegrated. The time required for full disintegration was recorded. Similarly, an identical set of three tubes, three alginate spheres per tube, were agitated by vortex for a period of twenty minutes to gain a rough assessment of the product's mechanical stability without the use of ultrasound disruption.

\section{RESULTS}

Table 1. Calcium Carbonate Concentrations in 1\% Algin Related to Time to Total Disruption with 85 Watt Ultrasound in 1 Second Bursts

\begin{tabular}{llll}
\hline & Trial One & Trial Two & Trial Three \\
\hline $50 \% \mathrm{CaCO}_{3}$ & $16: 34$ & $19: 43$ & $19: 22$ \\
\hline $75 \% \mathrm{CaCO}_{3}$ & $11: 45$ & $15: 55$ & $16: 22$ \\
\hline $100 \% \mathrm{CaCO}_{3}$ & $8: 45$ & $12: 10$ & $9: 12$ \\
\hline
\end{tabular}

Using the same parameters of three particles per plastic tube and 85 Watt ultrasound bursts of 1 second on/off, the time necessary to disintegrate the particles was found as a function of calcium carbonate concentration in the initial encapsulation mixture (see Table 1). Three trials at each concentration were used. Disintegration of the spheres was determined qualitatively by viewing the attained product under a microscope.

Table 2. Spheres Subjected to Agitation Via Vortex

\begin{tabular}{llll}
\hline & Trial One & Trial Two & Trial Three \\
\hline $50 \% \mathrm{CaCO}_{3}$ & $>30 \mathrm{~min}$ & $>30 \mathrm{~min}$ & $>30 \mathrm{~min}$ \\
\hline $75 \% \mathrm{CaCO}_{3}$ & $>30 \mathrm{~min}$ & $>30 \mathrm{~min}$ & $>30 \mathrm{~min}$ \\
\hline $100 \% \mathrm{CaCO}_{3}$ & $>30 \mathrm{~min}$ & $>30 \mathrm{~min}$ & $>30 \mathrm{~min}$ \\
\hline
\end{tabular}

Similarly, three spheres of each of the three calcium carbonate concentrations were subjected to three trials of agitation by vortex. In each case, no signs of particle disintegration could be observed after thirty minutes (see Table 2).

\section{DISCUSSION}

It is clear that an increasing concentration of calcium carbonate leads to a decrease in the time necessary for particle disintegration. Increasing the concentration of calcium carbonate both raises the amount of material in each sphere sensitive to ultrasound and reduces the relative concentration of alginate. As such there becomes less alginate hydrogel to hold particles together. We experimented with a range of values before running final trials at $50 \%, 75 \%$ and $100 \%$ calcium carbonate. Using calcium carbonate concentrations of less than $50 \%$ drastically increased the time necessary for ultrasound disruption, while concentrations greater than $100 \%$ increased viscosity of the mixture and made extrusion through the needle very difficult. 
Time-dependent diffusion of substances through an alginate matrix has been previously demonstrated, with diffusion times varying over minutes to hours depending upon the weight of the molecule in question and the initial concentration of sodium alginate used to make an encapsulation polymer (10). While we may reasonably conjecture that the particles generated in this study would share diffusion characteristics with those published, our initial goal was to dry particles in order to create a hard external calcium carbonate diffusion barrier. Drying particles in an incubator, however, generated a product that adhered too firmly to the drying surface to be removed without damage. A number of drying surfaces were attempted without success.

Limitations of this study include the lack of a quantitative method to determine the rate of sphere disintegration as a function of the number of ultrasound bursts to which they were subjected. Second, a method to quantify the amount of agitation delivered would be helpful so that the maximum mechanical disruption tolerated by spheres can be identified. Finally, although the coaxial airflow technique described in this study is a simple and widely used system of generating even micro-scale hydrogel particles, we found the reproducibility of particle shape and size to be limited from batch to batch. There are techniques available which offer greater reproducibility of product and smaller sphere size, albeit at much greater cost (11).

\section{CONCLUSION}

We have demonstrated a process by which routinely available and non-toxic materials can be used to create particles of encapsulated calcium carbonate in a calcium alginate hydrogel. The resulting product is easily disrupted with ultrasound and yet retains structural integrity under agitation by vortex for an extended period. There are logical implications for these particles to be injected into a target tissue under image guidance and subsequently identified via fluoroscopy or CT scan. Destruction of particles with therapeutic ultrasound and the controlled release of encapsulated drugs is a reasonable future experimental goal.

Our next set of experiments will involve a system to dry spheres while they are simultaneously agitated. It will thus be possible to quantify the diffusion characteristics and parameters of ultrasound disruption on dried particles. We are also working with microscopes that can photograph particles, the disruption products, as well as potentially measure the reduction of particle radius as a function of ultrasound delivery.

\section{ACKNOWLEDGEMENTS}

The authors would like to thank Dr. Aaron Fenster and Dr. Donald Downey from the Robarts Imaging Group at the University of Western Ontario for their meetings with us and expert advice. We also extend thanks to Dr. Argyrios Margaritis from the Department of Chemical and Biochemical Engineering at the University of Western Ontario for his expertise in encapsulation technology and assistance with equipment construction.

\section{REFERENCES}

1. Richard A, Margaritis A. Production and Mass Transfer Characteristics of Non-Newtonian Biopolymers for Biomedical Applications. Critical Reviews in Biotechnology 22(4): 355$374 ; 2002$.

2. Chemical Market Reporter, Drug Delivery Market Poised for Five Years of Strong Growth. Schnell Publishing, New York, 258(23); 2000.

3. Sershen S, West J. Implantable Polymeric Systems for Modulated Drug Delivery. Advanced Drug Delivery Reviews 54:1225$1235 ; 2002$.

4. Kost J, Wolfrum J, Langer R. Magnetically Enhanced Insulin Release in Diabetic Rats. Journal of Biomedical Materials Research 21: 1367-1373; 1987.

5. Kwok C, Mourad PD, Crum LA, Ratner BD. Self-Assembled Molecular Structures as Ultrasonically-Responsive Barrier Membranes for Pulsatile Drug Delivery. Journal of Biomedical Materials Research 57(2): 151-164; 2001.

6. Dinarvand R, D'Emanuele A. The Use of Thermoresponsive Hydrogels for On-Off Release of Molecules. Journal of Controlled Release 36: 221-227; 1995.

7.Fiszman GL, Karara, AL, Finocchiaro, LME, Glikin GC. A Laboratory Device for Microencapsulation of Genetically Engineered Cells into Alginate Beads. Electronic Journal of Biotechnology 5(3); 2002.

8. Mitri FG, Fellah ZEA, Closset E, Trompette P, Chapelon JY. Determination of Object Resonances by Vibro-Acoustography and their Associated Modes. Ultrasonics 42(1-9): 537-543; 2004.

9. Yang H, Wright JR. Calcium Alginate. In: Kuhtreiber WM, Lanza RP, Chick WL. Cell Encapsulation Technology and Therapeutics. Berlin, Germany: Birkhauser Publishing, 1999.

10. Millman JS. Diffusivity Characteristics of Globular Proteins in Calcium Alginate Immobilization Matrices. Faculty of Graduate Studies, University of Western Ontario, London, ON, 1992.

11. Dulieu C, Poncelet D, Neufeld R. Encapsulation and Immobilization Techniques. In: Goosen MFA, editor. Fundamentals of Animal Cell Encapsulation. Anne Arbor, MI: CRC Press, 1993.

Brent Lanting graduated from the Schulich School of Medicine in 2006 and will enter a residency in Orthopedic Surgery in London University with the eventual goal of a career in academic orthopedics.

Joe Barfett graduated from the Schulich School of Medicine in 2006 and will enter University of Toronto's Medical Imaging program with the hope of pursuing a career in clinical science. 